Nervenarzt 2008 79 [Suppl 4]:177

DOI 10.1007/s00115-008-2612-1

(C) Springer Medizin Verlag 2008

\title{
Sehr geehrte Kolleginnen und Kollegen,
}

ist auch die Vermeidung von Schnittstellenproblemen bei der Versorgung im sektorisierten Versorgungssystem neu zu regeln, wobei die derzeitigen Modelle der Integrierten Versorgung erste Anhaltspunkte liefern können.

Neben diesen klinisch-praktischen Aspekten geht es aber auch um die Konzepte psychischer Störungen, die letzten Endes die Grundlagen von Diagnostik und Therapie im Fachgebiet Psychiatrie und Psychotherapie sind. Grenzziehungen von „noch gesund“ und „schon krank“ spielen bei den gegenwärtigen Diskussionen um die Einbeziehung „dimensionaler“ Diagnosekriterien im Prozess der Revision von ICD-10 und DSM-IV eine Rolle. Neue ethische Fragen stellen sich, wenn es darum geht, die „Leistungsfähigkeit“ auch Gesunder durch den Einsatz von Psychopharmaka oder Stimulantien zu „optimieren“. Doch ist dafür der Psychiater zuständig?

Das Leitthema der DGPPN Kongresse in den Jahren 2007 und 2008 ist folgerichtig die „Identität des Fachgebietes Psychiatrie und Psychotherapie“. Im diesjährigen Kongress lautet das Schwerpunktthema „Die Psychiatrie als therapeutische Disziplin“. In der Psychopharmakologie werden neue Wirkprinzipien in klinischen Studien erforscht, so in der Schizophrenie-Behandlung die Beeinflussung von Glutamat-Rezeptoren oder in der Alzheimer-Behandlung die Etablierung von Immunisierungsstrategien gegen die Ablagerung von Amyloid-Plaques. Die Psychotherapie-Forschung beschäftigt sich mit den neurobiologischen Grundlagen von Therapieeffekten, wobei häufig - aber nicht immer - in funktionellen Kernspintomographie-Untersuchungen die gleichen Regionen mit Durchblutungsveränderungen gefunden werden wie beim
Einsatz von Psychopharmaka. Aber bedeutet dies auch lokal-spezifische Effekte der eingesetzten Therapieverfahren? Oder zeigt dies nur die „gemeinsame Endstrecke" an, ohne dass hier der Wirkort zu finden wäre? Vielleicht können neue somatotherapeutische Verfahren wie die transkranielle Magnetstimulation oder die tiefe Hirnstimulation künftig auch Beiträge zur Pathophysiologie psychischer Störungen liefern.

Neben diesen grundlagenbezogenen Aspekten spielt auch die Versorgung von Menschen mit psychischen Störungen eine wichtige Rolle im DGPPN Kongress 2008. Dabei werden Fragen neuer Versorgungsmodelle ebenso zu diskutieren sein wie die sektorübergreifende Qualitätssicherung und die Frage der Bewertung von Therapieverfahren im Sinne der Kosten-Nutzen-Analyse.

Um bei der Vielzahl innovativer Themen Schwerpunkte zu setzen, wurden die folgenden fünf Hauptthemen benannt:

- Entwicklungsperspektiven psychiatrischpsychotherapeutischer Therapie

- Wissenschaftliche Fundierung psychiatrisch-psychotherapeutischer Therapieverfahren

- Innovative Versorgungsmodelle in Psychiatrie und Psychotherapie

- Die Stellung von Psychiatrie und Psychotherapie im Versorgungssystem

- Sektorübergreifende Qualitätssicherung

Der vorliegende Abstractband möge mit seinen Informationen zu den einzelnen Beiträgen die Auswahl aus dem vielfältigen Angebot erleichtern.

\section{Univ.-Prof. Dr. med. Wolfgang Gaebel} Kongresspräsident

Präsident der Deutschen Gesellschaft für Psychiatrie, Psychotherapie und Nervenheil- kunde
Implementierung der wissenschaftlichen Leitlinien der Fachgesellschaften. Dabei 